\title{
On volume-source representations based on the representation theorem
}

\author{
Mie Ichihara ${ }^{1 *}+\mathbb{D}$, Tetsuya Kusakabe ${ }^{1 \dagger}$, Nobuki Kame ${ }^{1}$ and Hiroyuki Kumagai²
}

\begin{abstract}
We discuss different ways to characterize a moment tensor associated with an actual volume change of $\Delta V{ }^{C}$, which has been represented in terms of either the stress glut or the corresponding stress-free volume change $\Delta V^{\top}$. Eshelby's virtual operation provides a conceptual model relating $\Delta V^{C}$ to $\Delta V^{T}$ and the stress glut, where non-elastic processes such as phase transitions allow $\Delta V^{T}$ to be introduced and subsequent elastic deformation of $-\Delta V^{T}$ is assumed to produce the stress glut. While it is true that $\Delta V^{T}$ correctly represents the moment tensor of an actual volume source with volume change $\Delta V^{C}$, an explanation as to why such an operation relating $\Delta V^{C}$ to $\Delta V^{\top}$ exists has not previously been given.

This study presents a comprehensive explanation of the relationship between $\Delta V^{C}$ and $\Delta V^{\top}$ based on the representation theorem. The displacement field is represented using Green's function, which consists of two integrals over the source surface: one for displacement and the other for traction. Both integrals are necessary for representing volumetric sources, whereas the representation of seismic faults includes only the first term, as the second integral over the two adjacent fault surfaces, across which the traction balances, always vanishes. Therefore, in a seismological framework, the contribution from the second term should be included as an additional surface displacement. We show that the seismic moment tensor of a volume source is directly obtained from the actual state of the displacement and stress at the source without considering any virtual non-elastic operations. A purely mathematical procedure based on the representation theorem enables us to specify the additional imaginary displacement necessary for representing a volume source only by the displacement term, which links $\Delta V^{C}$ to $\Delta V^{T}$. It also specifies the additional imaginary stress necessary for representing a moment tensor solely by the traction term, which gives the "stress glut." The imaginary displacement-stress approach clarifies the mathematical background to the classical theory.
\end{abstract}

Keywords: Moment tensor, Volume source, Volcano seismology

\section{Background}

A moment tensor inversion is a powerful tool for extracting source information from seismic and geodetic observations. Volumetric changes are often related to the movement and expansion of fluid (i.e., magma and gas) at active volcanoes (Chouet 1996; Nishimura et al. 2000; Kazahaya et al. 2011; Maeda and Takeo 2011) or to underground explosions (Richards and Kim 2005). Estimating the amount of fluid mass involved in a deformation

\footnotetext{
*Correspondence: ichihara@eri.u-tokyo.ac.jp

${ }^{\dagger}$ Equal contributors

1 Earthquake Research Institute, The University of Tokyo, Yayoi, Bunkyo-ku,

Tokyo 113-0032, Japan

Full list of author information is available at the end of the article
}

event is a key for understanding volcanic processes. However, the interpretation of a moment tensor in terms of the actual deformation of the source region is non-unique (Backus and Mulcahy 1976; Wielandt 2003; Ampuero and Dahlen 2005). This uncertainty is more significant in the case of a source involving volumetric change and is therefore a critical issue in studies of volcanoes (Amoruso and Crescentini 2009; Kumagai et al. 2014; Mizuno et al. 2015). Because of the uncertainty, the source process cannot be directly determined by the seismic source inversion. Alternatively, fluid mechanical models for the source process can be compared with the seismic observation, if the geometry, volume change, and stress change in the source are linked to a seismic moment.

\section{至}


The current way of connecting a source volume change to a seismic moment tensor is not straightforward. The seismic moment of a spherical source has been defined in two different ways (Müller 2001; Richards and Kim 2005):

$$
\begin{aligned}
& M_{0}=(\lambda+2 \mu) \Delta V^{C}, \\
& M_{0}=(\lambda+2 \mu / 3) \Delta V^{T},
\end{aligned}
$$

where $\lambda$ and $\mu$ are Lamé's constants and $\Delta V^{C}$ and $\Delta V^{T}$ are two different definitions of a source volume change. $\Delta V^{T}$ is directly obtained under the assumption of a moment tensor for an internal surface characterized by a displacement gap, and $\Delta V^{C}$ is the actual volume change at the source. The difference between $\Delta V^{T}$ and $\Delta V^{C}$ has been discussed by various authors; for a review, see Kumagai et al. (2014). The two volumes are connected with each other through Eshelby's virtual operation approach (Eshelby 1957) by Aki and Richards (1980, 2002). Figure 1 summarizes this operation. First, the source material is separated by cutting along a closed surface that surrounds the source (Fig. 1a). Then, it is removed from the matrix and undergoes an inelastic (stress-free) deformation by $\Delta V^{T}$ (Fig. 1b). The inelastically deformed source volume is elastically strained and pushed back to its original place: this will generate an additional stress field $\Delta \sigma^{T}$ in the source volume and an additional traction is applied on its surface to hold its shape (Fig. 1c). Finally, the source material is welded across the cut surface, and the applied traction is released. Both the source and matrix deform elastically, which results in the source volume change of $\Delta V^{C}$ with the elastic stress change from $\Delta \sigma^{T}$ to $\Delta \sigma^{C}$ (Fig. 1d).

Eshelby's approach was originally introduced to obtain the equivalent body force, or the "stress glut," in the moment tensor representation of a volume source. The stress glut is defined as the difference between the true physical stress and the model stress (Backus and Mulcahy 1976) and has been shown to be equivalent to the moment-density tensor (Backus and Mulcahy 1976; Aki and Richards 1980). In Eshelby's model, the stress glut is given by the stress $\Delta \sigma^{T}$ in the source material after pushed back into its original space under elastic strain (Fig. 1c). Consequently, writing the true stress as $\Delta \sigma^{C}$
(Fig. 1d), the model stress (Backus and Mulcahy 1976) is $-\left(\Delta \sigma^{T}-\Delta \sigma^{C}\right)$, which is tensional in the case shown in Fig. 1.

The actual changes (i.e., $\Delta V^{C}$ and the actual pressure change) are relevant in the fluid mechanical modeling for the source, while the moment tensor representation is directly linked to the effective values (i.e., $\Delta V^{T}$ and the stress glut). Defining the relationship between the actual changes and the moment tensor representation is known to be a potentially confusing problem (Aki and Richards 1980; Aki and Richards 2002). The moment tensor representation of a spherical volume source in terms of the stress glut, which in this isotropic case is the pressure glut $\Delta p$, was originally given by equation 3.34 of Aki and Richards (1980) as

$$
M=\frac{4 \pi}{3} a^{3}\left(\begin{array}{ccc}
\Delta p & 0 & 0 \\
0 & \Delta p & 0 \\
0 & 0 & \Delta p
\end{array}\right)
$$

along with the remark "in the above equation, $\Delta p$ should not be confused with the pressure jump at the spherical surface at radius a." The expression given in (3) was replaced in the second edition of their textbook (equation. 3.35 in Aki and Richards, 2002) by

$M=\left(\begin{array}{ccc}\left(\lambda+\frac{2}{3} \mu\right) \Delta V^{T} & 0 & 0 \\ 0 & \left(\lambda+\frac{2}{3} \mu\right) \Delta V^{T} & 0 \\ 0 & 0 & \left(\lambda+\frac{2}{3} \mu\right) \Delta V^{T}\end{array}\right)$,

with the comment that "in the above equation, $\Delta V^{T}$ is the stress-free volume change and should not be confused with the volume change $\Delta V^{C}$ of a confined source region."

It is commonly accepted that the volume change directly related to the isotropic part (i.e., the trace) of the moment tensor is $\Delta V^{T}$, given by the equation

$$
\operatorname{tr}(M)=3\left(\lambda+\frac{2}{3} \mu\right) \Delta V^{T}
$$

and that $\Delta V^{C}$ can be obtained from $\Delta V^{T}$ by Eshelby's operation. However, it can be difficult to understand the mathematical reasoning underpinning this calculation, because Eshelby's operation is entirely conceptual
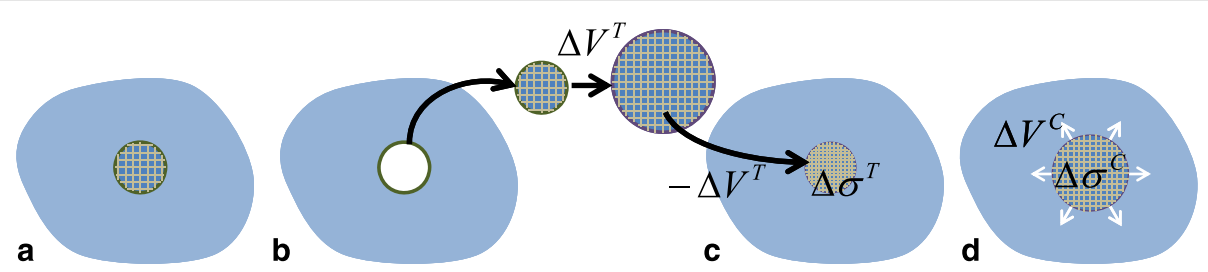

Fig. 1 Schematic illustration of Eshelby's operation, which consists of cutting $(\mathbf{a} \rightarrow \mathbf{b})$, a stress-free transformation by $\Delta V^{\top}(\mathbf{b})$, elastic straining $(\mathbf{c})$, and welding (d). The width of the mesh in the inner body indicates the extent of elastic compression or expansion, i.e., a finer or coarser mesh is associated with a higher or lower pressure, respectively 
and assumes specific processes for the stress-free volume change, such as phase transition.

Richards and Kim (2005) noted this problem for the case of the moment tensor representation of an underground explosion. The use of the definition of $\Delta V^{T}$ may appeal, since it is associated with a stress-free strain that phenomenologically appears to describe the consequences of the explosion in an unconfined volume, which is determined by the explosion yield. In reality, seismic moments differ for explosions having the same charge size placed at different depths. Therefore, $\Delta V^{T}$ is not literally a stressfree volume change of the charge excited by the explosion (Richards and Kim 2005). Kumagai et al. (2014) made comprehensive case studies of isotropic volume sources, finding different forms of the relation between $\Delta V^{T}$ and the source volume change $\Delta V^{C}$ depending on the source material and geometry.

Here, we present a straightforward explanation of the relationships among $\Delta V^{T}, \Delta V^{C}$, the stress glut, and the actual stress for a general volume source based solely on the representation theorem, which in turn yields the displacement field using Green's function. In the absence of body forces, the displacement field consists of two integrals over the source surface: one for surface displacement and the other for surface traction. First, we demonstrate that in contrast to the representation of earthquake shear faulting, the implicit assumption of omitting the surface traction integral, made while deriving the moment tensor, does not hold for a volume source. Second, we introduce a mathematical procedure developed in the boundaryintegral equation methods (Altiero and Gavazza 1980) for representing a volume source, which adequately considers the traction contribution. The traction correction turns out to be an additional surface displacement in the other surface integral term, causing an "imaginary volume change." We therefore propose an alternative definition of $\Delta V^{T}$ as the sum of $\Delta V^{C}$ and this imaginary volume change.

\section{Method}

\section{A conventional moment tensor representation in seismology}

We consider an elastic body with volume $V_{e x}$ and external surface $S$ and with internal surface $\Sigma$ of a volume source. The elastic deformation field in $V_{e x}$ is produced by the displacement and traction exerted on the surfaces $\Sigma$ and $S$ in the case of a no-body force. The representation theorem gives an expression for the displacement $u_{k}$ in $V_{e x}$ (Aki and Richards, 1980, equation 2.41):

$$
u_{k}(x, t)=-\int_{\Sigma+S} c_{i j p q} u_{i} n_{j} * G_{k p, q} d \Sigma+\int_{\Sigma+S} T_{i} * G_{k i} d \Sigma
$$

where $G_{k p}$ is the elastic Green's function in the medium without the internal surface $\Sigma, G_{k p, q}$ is its spatial derivative in the $q$ th direction, $c_{i j p q}$ is the elastic constant tensor, $T_{i}$ is the traction, and $n_{j}$ is a unit vector normal to the surface and directed outwards from $V_{e x}$. It is noted that $n_{j}$ on the internal surface $\Sigma$ is customarily taken outwards from $V_{e x}$. The surface integral on $S$ in (6) can be eliminated if we assume that both $u_{i}$ and $G_{k i}$ satisfy the same homogeneous boundary conditions on $S$; otherwise, we have to exactly evaluate the integral on $S$ (Kame and Kusakabe 2012). We note that $f * g=\int_{0}^{t} f(\tau) g(t-\tau) d \tau=$ $\int_{0}^{t} f(t-\tau) g(\tau) d \tau=\int_{-\infty}^{\infty} f(\tau) g(t-\tau) d \tau$ if $f(t)$ and $g(t)$ are zero for $t<0$ (Aki and Richards 1980). Under the assumption of homogeneous boundary conditions on $S$, the first and second terms of (6) represent contributions due to the displacement and traction on $\Sigma$, respectively.

In the case of a seismic source due to faulting, $\Sigma$ consists of two adjacent surfaces, labeled $\Sigma^{+}$and $\Sigma^{-}$, which are the opposing faces of the fault, in which case (6) is rewritten as

$$
u_{k}(x, t)=\int_{\Sigma} c_{i j p q}\left[u_{i}\right] n_{j} * G_{k p, q} d \Sigma-\int_{\Sigma}\left[T_{i}\right] * G_{k i} d \Sigma,
$$

where square brackets are used to denote the difference between the enclosed values on $\Sigma^{+}$and $\Sigma^{-}$and the surface-normal vector on $\Sigma$ is taken in the direction from $\Sigma^{-}$to $\Sigma^{+}$following the convention adopted by Aki and Richards (1980). It is the same direction as the normal vector on $\Sigma^{-}$, that is, opposite to the original surface-normal vector on $\Sigma$. That is why Eq. (7) has an opposite sign from (6). The continuity of traction across the fault surfaces means that $\left[T_{i}\right]$ is always zero; consequently, (7) reduces to

$$
u_{k}(x, t)=\int_{\Sigma} c_{i j p q}\left[u_{i}\right] n_{j} * G_{k p, q} d \Sigma,
$$

and the moment-density tensor $m_{p q}$ is defined in terms of the displacement discontinuity as

$$
m_{p q}=c_{i j p q}\left[u_{i}\right] n_{j} \text {. }
$$

When a fault plane $\Sigma$ is effectively regarded as a point source, (8) is approximated by

$$
\begin{aligned}
u_{k}(x, t) & =M_{p q} * G_{k p, q}, \\
M_{p q} & =\int_{\Sigma} m_{p q} d \Sigma,
\end{aligned}
$$

where $M_{p q}$ is the moment tensor.

The elastic tensor for an isotropic body is

$$
c_{i j p q}=\lambda \delta_{i j} \delta_{p q}+\mu\left(\delta_{i p} \delta_{j q}+\delta_{i q} \delta_{j p}\right),
$$

where $\lambda$ and $\mu$ are Lamé parameters and $\delta_{i j}$ is the Kronecker symbol. Equation (12) reduces (9) to

$$
m_{p q}=\lambda\left[u_{i}\right] n_{i} \delta_{p q}+\mu\left(\left[u_{q}\right] n_{p}+\left[u_{p}\right] n_{q}\right) .
$$




\section{Volumetric source representations in seismology}

The preceding discussion is not limited to a shear fault but includes opening on the fault, i.e., a displacement discontinuity oriented perpendicular to the fault surface. Let us now consider the case where a surface $\Sigma=\Sigma^{+}+\Sigma^{-}$ lies on the $x_{3}=0$ plane and the displacement discontinuity has a uniform non-zero component solely in the $x_{3}$-direction perpendicular to $\Sigma$. This source corresponds to an opening crack, and it represents a typical example of a volume source. For (11), the moment tensor representation of a thin crack with an opening dislocation in an isotropic medium is

$$
M=\left(\begin{array}{ccc}
\lambda & 0 & 0 \\
0 & \lambda & 0 \\
0 & 0 & \lambda+2 \mu
\end{array}\right)\left[u_{3}\right] \Sigma .
$$

An isotropic volume source may be represented by three perpendicular planar cracks with equal area $\Sigma$ and opening $[u]$, given

$$
M=\left(\begin{array}{ccc}
\lambda+\frac{2}{3} \mu & 0 & 0 \\
0 & \lambda+\frac{2}{3} \mu & 0 \\
0 & 0 & \lambda+\frac{2}{3} \mu
\end{array}\right)(3[u] \Sigma) .
$$

Note that (15) corresponds to (4), if the total opening volume $3[u] \Sigma$ is the stress-free volume change $\Delta V^{T}$.

For the isotropic moment tensor in (15), the 3D static Green's function directly gives the radial displacement field $u_{r}(x)=u_{r}^{\text {iso }}(r)$ as a function of distance $r$ from the center of the cracks as follows (Kumagai et al. 2014):

$$
u_{r}^{\text {iso }}(r)=\frac{\lambda+\frac{2}{3} \mu}{\lambda+2 \mu} \frac{\Delta V^{T}}{4 \pi r^{2}},
$$

noting that only a radial displacement exists. On the other hand, the analytic displacement field, as obtained for a spherically expanding volume source by Kumagai et al. (2014), is

$$
u_{r}^{\text {sol }}=\frac{\Delta V^{C}}{4 \pi r^{2}},
$$

where $\Delta V^{C}$ is the actual volume change in the source region.

If $\Delta V^{T}$ in (16) is equal to the spherical volume change $\Delta V^{C}$ in (17), the radial displacement $u_{r}^{\text {iso }}$ in (16) is smaller than $u_{r}^{\text {sol }}$ in (17) and the difference is

$$
u_{r}^{\text {sol }}-u_{r}^{\text {iso }}=\frac{\frac{4}{3} \mu}{\lambda+2 \mu} \frac{\Delta V^{C}}{4 \pi r^{2}} .
$$

Both displacements are identical if we assume

$$
\Delta V^{T}=\frac{\lambda+2 \mu}{\lambda+\frac{2}{3} \mu} \Delta V^{C},
$$

and this adjustment factor is the same as that conventionally adopted. In the literature, the adjustment has been explained with reference to Eshelby's operation (Eshelby 1957) assuming $\Delta V^{T}$ is the stress-free volume change
(Aki and Richards 2002). Müller (2001) considered (15) to be true when the isotropic source consists of opening cracks that are homogeneously distributed over the surface of a sphere, in which case $\Delta V^{T}$ is the total opening while $\Delta V^{C}$ is the fraction that opens outward. Although it has been acknowledged that the adjustment for a spherical volumetric source is correct, a method for obtaining the representation from the seismological moment tensor has not been clearly explained for the general case.

Here, we note that the difference in (19) originates from the second term of (6) that is not included for seismic fault sources. The first and second surface integral terms of (6) respectively give

$$
\begin{aligned}
& u_{r}^{\mathrm{dis}}=\frac{\lambda+\frac{2}{3} \mu}{\lambda+2 \mu} \frac{\Delta V^{C}}{4 \pi r^{2}}, \\
& u_{r}^{\mathrm{tra}}=\frac{\frac{4}{3} \mu}{\lambda+2 \mu} \frac{\Delta V^{C}}{4 \pi r^{2}} .
\end{aligned}
$$

By summing $u_{r}=u_{r}^{\text {dis }}+u_{r}^{\text {tra }}$, we directly obtain the analytical solution (17) without adjusting the volume change; the mathematical procedure required to obtain this result is given in the Appendix.

Although both terms of (6) are necessary to fully represent the displacement field with the true boundary conditions, it is commonly inconvenient to include two terms in wave analyses. In the following section, we show that using the stress-free volume change, or the stress glut, provides a way of combining the two terms into a single term.

\section{Volume-source representations based on the representation theorem}

We consider the displacement field due to the expansion of a source cavity bounded by $\Sigma$ within a surrounding elastic body (see Fig. 3a, b). The displacement field in the elastic body is then represented only by integration on $\Sigma$ as per (6) with the specified Green's function, regardless of the material inside the source body. Here, it is assumed that the displacement and the traction on $\Sigma$ are specified. Note that these quantities are not independent but should obey the elastic equations.

First, we replace the material inside the cavity with an elastic body $V_{\text {in }}$ with surface $\Sigma_{i n}$ that has the same mechanical properties as the surrounding elastic body and the same shape as the cavity. It is assumed that this procedure does not change the boundary conditions on $\Sigma$ so that the deformation field $u_{n}$ is unchanged. The normal vector for $\Sigma_{\text {in }}$ is directed outwards with respect to $V_{\mathrm{in}}$, the opposite direction to that for $\Sigma$. The representation (6) may include the surface integral on $\Sigma_{i n}$ :

$$
u_{k}(x, t)=-\int_{\Sigma+\Sigma_{i n}} c_{i j p q} u_{i} n_{j} * G_{k p, q} d \Sigma+\int_{\Sigma+\Sigma_{i n}} T_{i} * G_{k i} d \Sigma .
$$


Because $V_{\text {in }}$ is composed of the same elastic material as the external body, the elastic deformation field in $V_{\text {in }}$, including the traction and displacement relation on $\Sigma_{i n}$, satisfies the same elastic equations so that the same Green's function is applicable on $\Sigma_{i n}$. Moreover, Betti's theorem guarantees that

$-\int_{\Sigma_{i n}} c_{i j p q} u_{i} n_{j} * G_{k p, q} d \Sigma+\int_{\Sigma_{i n}} T_{i} * G_{k i} d \Sigma=0$,

outside $V_{\text {in }}$. Because $\Sigma$ and $\Sigma_{i n}$ are the same, but opposing, faces of the cavity surface, the integrals on the two surfaces are combined in the same way as (7), giving

$$
u_{k}(x, t)=\int_{\Sigma} c_{i j p q}\left[u_{i}\right] n_{j} * G_{k p, q} d \Sigma-\int_{\Sigma}\left[T_{i}\right] * G_{k i} d \Sigma,
$$

where the direction of the surface-normal vector on $\Sigma$ is replaced by $n_{j}^{-}$that is directed from $\Sigma_{i n}$ to $\Sigma$ (Fig. 2) following Aki and Richards (1980) as in Eq. (7). Adequately taking $\left[u_{i}\right]$ and $\left[T_{i}\right]$ allows the displacement field to be represented either by the first or the second integral term on the right-hand side of Eq. (24), which is the so-called indirect boundary-integral equation method (Altiero and Gavazza 1980).

When the body $V_{\text {in }}$ is subject to the same surface traction $T_{i}^{C}$ on its surface $\Sigma_{i n}$ as that on $\Sigma$, the term with $\left[T_{i}\right]$ vanishes in (24) and we obtain the same representation as (8). The elastic displacement on $\Sigma_{\text {in }}$ is not the same as that on $\Sigma$ but is the value bringing $V_{\text {in }}$ from zero traction to the given traction by elastic deformation (Fig. 3c). Therefore, the displacement discontinuity $\left[u_{i}\right]$ in $(8)$ is interpreted as the difference between the actual displacement $u_{i}^{C}$ on the cavity wall and the imaginary displacement $u_{i}^{I}$ on $\Sigma_{i n}$ required to replace the traction term. It is also noted that $\left[u_{i}\right]$ corresponds to the difference between the surface of $V_{\text {in }}$ in the stress-free condition and the original size of the cavity and is therefore equivalent to the displacement in the stress-free transformation of Eshelby's operation (Fig. 1c).
Alternatively, we may eliminate the displacement term and keep the traction term in (24). In this case, we replace the material inside the cavity with an elastic body $V_{\text {in }}$ with surface $\Sigma_{\text {in }}$ that has the same mechanical properties as the surrounding elastic body and the same shape as the cavity. The surface displacement on $\Sigma_{i n}$ is assumed to be $u_{i}^{C}$ as that on $\Sigma$ (Fig. 3d). The deformation of $V_{\text {in }}$ under this boundary condition should satisfy the elastic equations as in the previous case. The surface traction on $\Sigma_{i n}$ is then different from $T_{i}^{C}$. Rather than (8), we may instead represent the solution by

$$
u_{k}(x, t)=-\int_{\Sigma}\left[T_{i}\right] * G_{k i} d \Sigma .
$$

The stress discontinuity $\left[T_{i}\right]$ is the difference between the actual traction on the cavity wall $T_{i}^{C}$ and the imaginary traction $T_{i}^{I}$ that is required to replace the displacement term in (24). Because $T_{i}^{C}-T_{i}^{I}=T_{i}^{C}+\left(-T_{i}^{I}\right),\left[T_{i}\right]$ has an additional traction $-T_{i}^{I}$ that is equivalent to the deformation of $V_{\text {in }}$ by $-u_{i}^{C}$. Therefore, [ $\left.T_{i}\right]$ corresponds to the traction of the state shown in Fig. 1c in Eshelby's operation. Putting $\left[T_{i}\right]=-\left(\Delta \tau_{i j} n_{j}\right)$, Eq. (25) is equivalent with equation (3.26) of Aki and Richards (1980) with $\Delta \tau_{i j}$ being the stress glut of Backus and Mulcahy (1976).

Our interpretation can be illustrated well using a simple analytic problem. Consider the case where the cavity is a sphere with radius $R$ and volume $V=\frac{4}{3} \pi R^{3}$, filled by fluid with an overpressure $\Delta P^{C}$. The corresponding surface displacement of the cavity is directed radially outward with $u_{r}^{C}=R \Delta P^{C} /(4 \mu)$, and the volume change is $\Delta V^{C}=$ $V \Delta P^{C} /\left(\frac{4}{3} \mu\right)$ (Kumagai et al. 2014). On the other hand, the displacement on $\Sigma_{i n}$ for the same overpressure is directed radially inward with $4 \pi R^{2} u_{r}^{I}=-V \Delta P^{C} /\left(\lambda+\frac{2}{3} \mu\right)$, giving $u_{r}^{I}=-4 \mu u_{r}^{C} /(3 \lambda+2 \mu)$. The displacement gap used in the moment tensor is then

$$
\left[u_{r}\right]=u_{r}^{C}-u_{r}^{I}=\frac{\lambda+2 \mu}{\lambda+\frac{2}{3} \mu} u_{r}^{C} .
$$

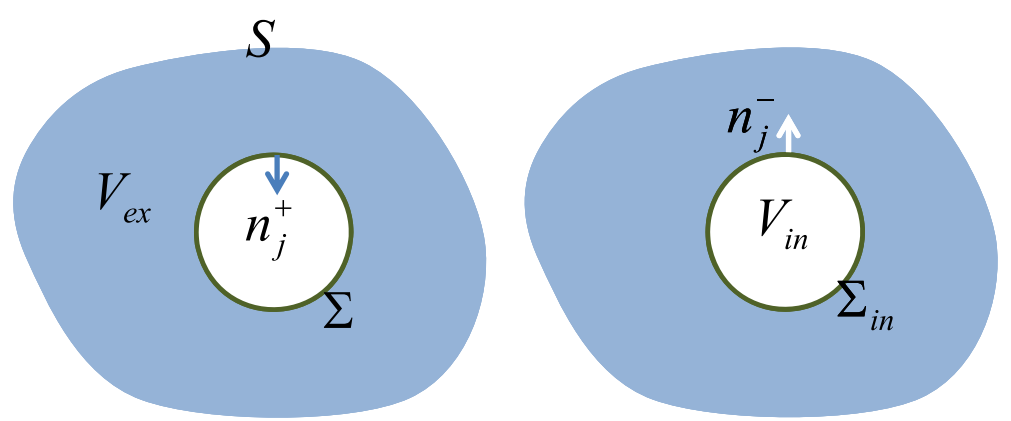

Fig. 2 A schematic illustration of the normal vectors. $n_{j}^{+}$and $n_{j}^{-}$on the internal surfaces $\Sigma$ and $\Sigma_{i n}$, respectively. $V_{\text {ex }}$ is the elastic medium of interest, and $V_{\text {in }}$ is the source volume 


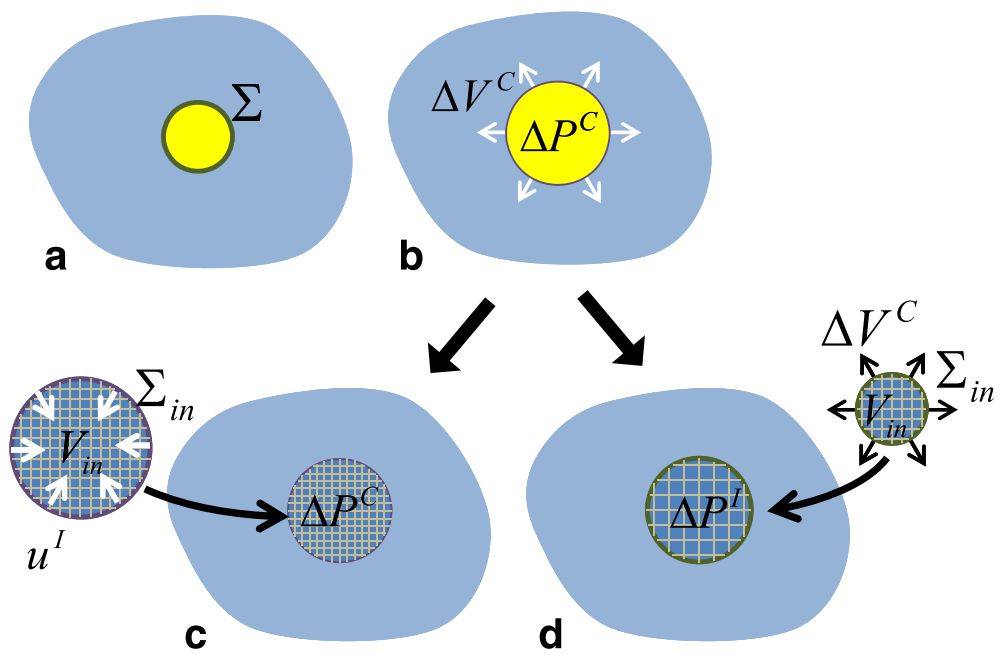

Fig. 3 Schematic illustration of our new interpretation: expansion from $\mathbf{a}$ to $\mathbf{b}$ by $\Delta V^{C}$ due to the overpressure $\Delta P^{C}$ in the fluid cavity bounded by $\Sigma$ drives the process. $\mathbf{c}$ The internal fluid is replaced by an elastic body $V_{\text {in }}$ consisting of the same elastic material as the external medium. $V_{\text {in }}$ has the same traction on $\Sigma_{i n}$ as the actual overpressure. The corresponding displacement on $\Sigma_{\text {in }}$ is $u_{i}^{\prime}$, which is different from the actual displacement on $\Sigma$. d The internal fluid is replaced by an elastic body $V_{\text {in }}$ consisting of the same elastic material as the external medium. A displacement identical to the actual displacement on $\Sigma(\mathbf{b})$ is applied to $V_{\text {in }}$. The pressure change in $V_{\text {in }}^{\prime}$ is then $\Delta P^{\prime}$, which differs from $\Delta P^{C}$

If we assume $\left[u_{r}\right]$ as the surface displacement, the corresponding volume change is

$$
4 \pi R^{2}\left[u_{r}\right]=\frac{\lambda+2 \mu}{\lambda+\frac{2}{3} \mu} \Delta V^{C},
$$

which is equal to Eshelby's stress-free volume change $\Delta V^{T}$ in (19). Alternatively, if the same outward displacement $u_{r}^{C}$ is applied to the inner sphere surface $\Sigma_{i n}$, the corresponding stress field in $V_{\text {in }}$ is a negative pressure defined as

$$
\Delta P^{I}=-\left(\lambda+\frac{2}{3} \mu\right) \frac{3 u_{r}^{C}}{R}=-\left(\lambda+\frac{2}{3} \mu\right) \frac{\Delta V^{C}}{V},
$$

and therefore,

$$
\left[T_{i}\right]=\left(\Delta P^{C}-\Delta P^{I}\right) n_{i}=(\lambda+2 \mu) \frac{\Delta V^{C}}{V} n_{i}=\left(\lambda+\frac{2}{3} \mu\right) \frac{\Delta V^{T}}{V} n_{i} .
$$

\section{Results and discussion}

\section{A unified explanation}

Here, we have shown the difference of the effective changes (i.e., $\Delta V^{T}$ and the stress glut) from the actual changes (i.e., $\Delta V^{C}$ and the actual pressure change) as a consequence of reducing the two surface integral terms in the representation (6) to one term as either (8) or (25). This reduction is done by replacing the surface displacement with $u_{i}^{C}-u_{i}^{I}$ or replacing the surface traction with $T_{i}^{C}-T_{i}^{I}$, respectively, which has already been shown by Altiero and Gavazza (1980) as the indirect boundaryintegral methods. Here, we make a link between their formulation and the moment tensor representation of a volume source. Given that the stress giving the traction $T_{i}^{C}-T_{i}^{I}$ corresponds to the so-called stress glut, we propose calling $u_{i}^{C}-u_{i}^{I}$ the "displacement glut," which is equivalent to Eshelby's stress-free transformation of Aki and Richards (2002) and to the spherical-crack opening of Müller (2001). We consider the term "displacement glut" to be more appropriate because it is not linked to specific processes such as the stress-free transformation or spherical-crack models and because it makes a logical counterpart to the stress glut. Moreover, the representation in terms of the displacement glut, rather than stress glut, is more useful because the seismic moment tensor inversion framework has been developed in the form of (8).

The present work generalizes the conclusion of Kumagai et al. (2014) for a bimaterial spherical source. In calculating the displacement glut or stress glut, it should be assumed that the internal body has the same elastic properties as the external body, regardless of the actual internal material. This understanding does not necessarily help in the interpretation of a moment tensor in terms of the physical source process, but it is useful for forward modeling from the source process to the equivalent moment tensor. When a source geometry is defined and $T_{i}^{C}$ or $u_{i}^{C}$ is specified on the source surface, $u_{i}^{I}$ or $T_{i}^{I}$ is obtained by solving the elastic equations for a body having the same geometry, the same traction, or displacement on its surface, and the same mechanical properties as those 
of the external elastic body. This calculation can be performed for arbitrary geometries. The distribution of $\left[u_{i}\right]$ on the surface can then be obtained, and the momentdensity tensor calculated by (9). Finally, the moment tensor of the equivalent point source is obtained by integrating the moment-density tensor over the source surface as in (11). This calculation can be performed using either numerical or analytical methods.

\section{Conclusion}

This work presents a comprehensive explanation as to why $\Delta V^{T}$, rather than $\Delta V^{C}$, appears in the moment tensor representation of a volumetric source. We mathematically redefine $\Delta V^{T}$ as the result of a displacement glut, instead of Eshelby's stress-free volume change concept. The difference between the displacement glut and the actual displacement of the source surface replaces the contribution of the traction surface integral term in the representation theorem, which has not previously been used in seismology. With this understanding, we can more confidently link the moment tensor representation with fluid mechanical modeling.

\section{Appendix}

Representation theorem for a spherical displacement field

We consider the static displacement field due to the expansion of a spherical cavity in an unbounded, isotropic, and homogeneous medium. The initial radius of the sphere is $R$. The origin of the spatial coordinate system is defined as the center of the sphere.

Green's function depends on the distance $L$ between the source at $\xi$ and the observation point at $x$ :

$$
\begin{aligned}
G_{k p}(L) & =\frac{1}{8 \pi \mu}\left(\delta_{k p} \frac{\partial^{2}}{\partial x_{j} \partial x_{j}}-\Gamma \frac{\partial^{2}}{\partial x_{k} \partial x_{p}}\right) L=\frac{1}{8 \pi \mu} D_{k p}^{x} L \\
D_{k p}^{x} & =\delta_{k p} \frac{\partial^{2}}{\partial x_{i} \partial x_{i}}-\Gamma \frac{\partial^{2}}{\partial x_{k} \partial x_{p}}, \\
\Gamma & =\frac{\lambda+\mu}{\lambda+2 \mu}, \\
L & =|x-\xi| .
\end{aligned}
$$

In the static case, the representation given by (6) can be rewritten:

$$
\begin{aligned}
u_{k}(x)= & -\int_{\Sigma} c_{i j p q} u_{i}(\xi) n_{j}(\xi) \frac{\partial G_{k p}(L)}{\partial \xi_{q}} d \Sigma(\xi) \\
& +\int_{\Sigma} T_{i}(\xi) G_{k i}(L) d \Sigma(\xi) .
\end{aligned}
$$

For clarity, in the following, we omit the dependence on the spatial variables where they are obvious from the context.
The first term is rewritten using (12) and separated into an isotropic part $u_{k}^{K u}$ and a non-isotropic part $u_{k}^{\mu u}$ :

$$
\begin{gathered}
u_{k}^{K u}=-K \int_{\Sigma} u_{i} n_{i} \frac{\partial G_{k p}}{\partial \xi_{p}} d \Sigma, K=\lambda+\frac{2}{3} \mu, \\
u_{k}^{\mu u}=-\mu \int_{\Sigma}\left[u_{i} n_{j}\left\{\frac{\partial G_{k i}}{\partial \xi_{j}}+\frac{\partial G_{k j}}{\partial \xi_{i}}\right\}-\frac{2}{3} u_{i} n_{i} \frac{\partial G_{k p}}{\partial \xi_{p}}\right] d \Sigma .
\end{gathered}
$$

The second term is represented by $u_{k}^{T}$ :

$$
u_{k}^{T}=\int_{\Sigma} T_{i} G_{k i} d \Sigma(\xi)
$$

Let both the displacement and traction on the sphere surface $\Sigma$ be directed radially outwards with magnitude $\Delta R$ and $\Delta P$, respectively:

$$
\begin{aligned}
& u_{i}(\xi)=-\Delta R n_{i}, \\
& T_{i}(\xi)=-\Delta P n_{i},
\end{aligned}
$$

where $n_{i}$ is the unit vector normal to the surface, pointing outwards from the elastic medium, i.e., into the sphere. We note that

$$
\Delta V^{C}=4 \pi R^{2} \Delta R
$$

and, according to the relation of linear elasticity,

$$
\Delta P=4 \mu \frac{\Delta R}{R} .
$$

Using the boundary conditions (38) and (39), Eqs. (35)(37) are rewritten as

$$
\begin{aligned}
& u_{k}^{K u}=K \Delta R \int_{\Sigma} \frac{\partial G_{k p}}{\partial \xi_{p}} d \Sigma, \\
& u_{k}^{\mu u}=2 \mu \Delta R \int_{\Sigma}\left[n_{i} n_{j} \frac{\partial G_{k i}}{\partial \xi_{j}}-\frac{1}{3} \frac{\partial G_{k p}}{\partial \xi_{p}}\right] d \Sigma, \\
& u_{k}^{T}=-\Delta P \int_{\Sigma} n_{i} G_{k i} d \Sigma .
\end{aligned}
$$

\section{The traction term}

We first evaluate (44) using Green's function (30):

$$
\begin{aligned}
u_{k}^{T} & =-\frac{\Delta P}{8 \pi \mu} \int_{\Sigma} n_{i}\left(\delta_{k i} \frac{\partial^{2}}{\partial \xi_{j} \partial \xi_{j}}-\Gamma \frac{\partial^{2}}{\partial \xi_{k} \partial \xi_{i}}\right) L d \Sigma \\
& =-\frac{\Delta P}{8 \pi \mu} D_{k i}^{x} \int_{\Sigma} n_{i} L d \Sigma,
\end{aligned}
$$

where we have used the relation $\partial L / \partial \xi_{k}=-\partial L / \partial x_{k}$ according to (33). Replacing the surface-normal vector $n_{i}$ by the opposite vector $v_{i}=-n_{i}$ directed outwards from 
the sphere, the surface integral in (45) is converted into a volume integral over the sphere $V_{\mathrm{s}}$, becoming

$$
\begin{aligned}
u_{k}^{T} & =\frac{\Delta P}{8 \pi \mu} D_{k i}^{x} \int_{\Sigma} v_{i} L d \Sigma=\frac{\Delta P}{8 \pi \mu} D_{k i}^{x} \int_{V_{\mathrm{s}}} \frac{\partial}{\partial \xi_{i}} L d V_{\mathrm{s}} \\
& =-\frac{\Delta P}{8 \pi \mu} D_{k i}^{x} \frac{\partial}{\partial x_{i}} \int_{V_{\mathrm{s}}} L d V_{\mathrm{s}} .
\end{aligned}
$$

Because the displacement field is spherically symmetric and non-directional, we may assume $x=(0,0, x)(x>0)$. Representing $\xi$ in a polar coordinate system, we have $L=$ $\sqrt{x^{2}+\xi^{2}-2 x \xi \cos \theta}$ and $d V=\xi^{2} d \xi \sin \theta d \theta d \phi$ and

$$
\begin{aligned}
u_{k}^{T}= & -\frac{\Delta P}{8 \pi \mu} D_{k i}^{x} \frac{\partial}{\partial x_{i}} \int_{0}^{R} d \xi \int_{0}^{2 \pi} d \phi \int_{o}^{\pi} \\
& \times d \theta \sin \theta \sqrt{x^{2}+\xi^{2}-2 x \xi \cos \theta} .
\end{aligned}
$$

Evaluating this integral, we obtain

$$
u_{k}^{T}=-\frac{\Delta P}{2 \mu} D_{k i}^{x} \frac{\partial}{\partial x_{i}}\left(\frac{R^{3}}{3} x+\frac{2 R^{5}}{15} \frac{1}{x}\right) .
$$

Recalling that $x$ represents $|x|=r$ and the definition of $D^{x}$ from (31), we have

$$
\begin{aligned}
& D_{k i}^{x} \frac{\partial}{\partial x_{i}}=(1-\Gamma) \frac{\partial}{\partial x_{k}} \frac{\partial^{2}}{\partial x_{i} \partial x_{i}} \\
& \frac{\partial^{2}}{\partial x_{i} \partial x_{i}} \frac{1}{r}=\frac{\partial}{\partial x_{i}}\left(-\frac{x_{i}}{r^{3}}\right)=-\frac{3}{r^{3}}+3 \frac{x_{i} x_{i}}{r^{5}}=0 \\
& \frac{\partial}{\partial x_{k}} \frac{\partial^{2}}{\partial x_{i} \partial x_{i}} r=\frac{\partial}{\partial x_{k}} \frac{\partial}{\partial x_{i}}\left(\frac{x_{i}}{r}\right)=\frac{\partial}{\partial x_{k}}\left(\frac{2}{r}\right)=-\frac{2}{r^{2}} \frac{x_{k}}{r} .
\end{aligned}
$$

Using (32) and (39), finally, we obtain the radial displacement in the same form as (21)

$$
u_{k}^{T}=\frac{4}{3} \mu \frac{\Delta V^{C}}{\lambda+2 \mu} \frac{x_{k}}{4 \pi r^{2}} .
$$

where $x_{k} / r$ is a unit vector in the radial direction.

\section{The isotropic part of the displacement term}

Equation (42) is calculated in a similar way as in the previous section:

$$
\begin{aligned}
u_{k}^{K u} & =K \Delta R \int_{\Sigma} \frac{\partial G_{k p}}{\partial \xi_{p}} d \Sigma=-\frac{K \Delta R}{8 \pi \mu} D_{k p}^{x} \frac{\partial}{\partial x_{p}} \int_{\Sigma} L d \Sigma \\
& =-\frac{\lambda \Delta R}{8 \pi \mu}(1-\Gamma) \frac{\partial}{\partial x_{k}} \frac{\partial^{2}}{\partial x_{p} \partial x_{p}} \int_{\Sigma} L d \Sigma
\end{aligned}
$$

Using a polar coordinate system and assuming $x=$ $(0,0, x)(x>0)$, we have

$$
\begin{aligned}
\int_{\Sigma} L d \Sigma & =R^{2} \int_{0}^{2 \pi} d \phi \int_{0}^{\pi} d \theta \sin \theta \sqrt{x^{2}+R^{2}-2 x R \cos \theta} \\
& =-2 \pi R^{2}\left(2 x+\frac{2 R^{2}}{3 x}\right) .
\end{aligned}
$$

The partial differentiation of (54) in (53) is conducted in the same way as in the previous subsection. Converting $\Delta R$ to $\Delta V^{C}$ using (40) and using the definition of $K(35)$, we obtain

$$
u_{k}^{K u}=\frac{\lambda+\frac{2}{3} \mu}{\lambda+2 \mu} \frac{\Delta V^{C}}{4 \pi r^{2}} \frac{x_{k}}{r} .
$$

The above expression for $u_{k}^{K u}$ gives the radial displacement in the same form as (20). In the following section, the remaining term $u_{k}^{\mu u}$ from (43) is shown to vanish.

\section{The non-isotropic part of the displacement term}

The values obtained for the first and second terms of the integrand in (43) are denoted as $u_{k}^{\mu u 1}$ and $u_{k}^{\mu u 2}$, respectively. The calculation of the latter follows the method outlined in the previous section, and it is obvious that

$$
u_{k}^{\mu u 2}=-\frac{\frac{2}{3} \mu}{\lambda+2 \mu} \frac{\Delta V^{C}}{4 \pi r^{2}} \frac{x_{k}}{r}
$$

the calculation of the first term is more complicated and is presented below.

$$
u_{k}^{\mu u 1}=2 \mu \Delta R \int_{\Sigma} n_{i} n_{j} \frac{\partial G_{k i}}{\partial \xi_{j}} d \Sigma=\frac{\Delta R}{4 \pi} D_{k i}^{x} \int_{\Sigma} n_{i} n_{j} \frac{\partial L}{\partial \xi_{j}} d \Sigma .
$$

Recalling the definition of $D_{k i}^{x}$ from (31), we further separate (57) into $A_{k}$ and $B_{k}$ as

$$
\begin{gathered}
A_{k}=\frac{\Delta R}{4 \pi} \frac{\partial^{2}}{\partial x_{p} \partial x_{p}} \int_{\Sigma} n_{k} n_{j} \frac{\partial L}{\partial \xi_{j}} d \Sigma, \\
B_{k}=-\frac{\Delta R}{4 \pi} \Gamma \frac{\partial^{2}}{\partial x_{k} \partial x_{i}} \int_{\Sigma} n_{i} n_{j} \frac{\partial L}{\partial \xi_{j}} d \Sigma .
\end{gathered}
$$


For mathematical convenience, the variable of partial differentiation in (59) is changed from $\partial / \partial x_{i}$ to $-\partial / \partial \xi_{i}$ to obtain

$$
\begin{aligned}
B_{k} & =\frac{\Delta R}{4 \pi} \Gamma \frac{\partial}{\partial x_{k}} \int_{\Sigma} n_{i} n_{j} \frac{\partial^{2} L}{\partial \xi_{i} \partial \xi_{j}} d \Sigma \\
& =\frac{\Delta R}{4 \pi} \Gamma \frac{\partial}{\partial x_{k}} \int_{\Sigma} n_{i} n_{j} \frac{\partial}{\partial \xi_{i}} \frac{\xi_{j}-x_{j}}{L} d \Sigma \\
& =\frac{\Delta R}{4 \pi} \Gamma \frac{\partial}{\partial x_{k}} \int_{\Sigma} n_{i} n_{j}\left[\frac{\delta_{i j}}{L}-\frac{\left(\xi_{j}-x_{j}\right)\left(\xi_{i}-x_{i}\right)}{L^{3}}\right] d \Sigma \\
& =\frac{\Delta R}{4 \pi} \Gamma \frac{\partial}{\partial x_{k}} \int_{\Sigma}\left[\frac{1}{L}-\frac{\left(\xi_{j}-x_{j}\right) n_{i}\left(\xi_{i}-x_{i}\right) n_{j}}{L^{3}}\right] d \Sigma .
\end{aligned}
$$

Returning to polar coordinates, we have $\int_{\Sigma} d \Sigma=$ $R^{2} \int_{0}^{2 \pi} d \phi \int_{0}^{\pi} d \theta \sin \theta$. We let $x=(0,0, x)(x>0)$, and

$$
n=\left(\begin{array}{c}
-\sin \theta \cos \phi \\
-\sin \theta \sin \phi \\
-\cos \theta
\end{array}\right)
$$

As in (54), we also represent $L$ in polar coordinates, from which we obtain

$$
n_{j} \frac{\partial L}{\partial x_{j}}=\frac{\left(\xi_{j}-x_{j}\right) n_{j}}{L}=\frac{-R+x \cos \theta}{L} .
$$

Substituting (61) and (62) into (58) and (60), and conducting the integration with respect to $\phi$, we obtain

$$
A=\frac{R^{2} \Delta R}{2} \frac{\partial^{2}}{\partial x_{p} \partial x_{p}} \int_{0}^{\pi} d \theta \sin \theta \frac{-R+x \cos \theta}{L}\left(\begin{array}{c}
0 \\
0 \\
-\cos \theta
\end{array}\right) \text {, }
$$

$$
\begin{aligned}
B_{k} & =\frac{R^{2} \Delta R}{2} \Gamma \frac{\partial}{\partial x_{k}} \int_{0}^{\pi} d \theta \sin \theta \frac{1}{L}\left[1-v \frac{(-R+x \cos \theta)^{2}}{L^{2}}\right] d \Sigma \\
& =\frac{R^{2} \Delta R}{2} \Gamma \frac{\partial}{\partial x_{k}} \int_{0}^{\pi} d \theta \sin \theta \frac{x^{2}\left(1-\cos ^{2} \theta\right)}{L^{3}} d \Sigma .
\end{aligned}
$$

From the definition of $L$, we have

$$
\cos \theta=\frac{L^{2}-x^{2}-R^{2}}{-2 x R}, \sin \theta d \theta=-d(\cos \theta)=\frac{L}{x R} d L .
$$

Therefore, (63) becomes

$$
\begin{aligned}
A= & \frac{R^{2} \Delta R}{2} \frac{\partial^{2}}{\partial x_{p} \partial x_{p}} \int_{|x-R|}^{|x+R|} d L \frac{L}{x R} \frac{R \cos \theta-x \cos ^{2} \theta}{L}\left(\begin{array}{l}
0 \\
0 \\
1
\end{array}\right) \\
= & \frac{R^{2} \Delta R}{2} \frac{\partial^{2}}{\partial x_{p} \partial x_{p}} \int_{|x-R|}^{|x+R|} d L \frac{R\left(\frac{L^{2}-x^{2}-R^{2}}{-2 x R}\right)-x\left(\frac{L^{2}-x^{2}-R^{2}}{-2 x R}\right)^{2}}{x R} \\
& \times\left(\begin{array}{l}
0 \\
0 \\
1
\end{array}\right),
\end{aligned}
$$

and (64) reduces to

$$
B_{k}=\frac{R^{2} \Delta R}{2} \Gamma \frac{\partial}{\partial x_{k}} \int_{|x-R|}^{|x+R|} d L \frac{x}{R L^{2}}\left[1-\left(\frac{L^{2}-x^{2}-R^{2}}{-2 x R}\right)^{2}\right] .
$$

We note that the solution outside the sphere is obtained when $|x-R|=x-R$ in (66) and (67), while that inside the sphere is obtained when $|x-R|=-x+R$.

Evaluating the algebra and integration with respect to $L$ in (66) and (67), we obtain

$$
\begin{aligned}
A & =\frac{R^{2} \Delta R}{2} \frac{\partial^{2}}{\partial x_{p} \partial x_{p}}\left(-\frac{2}{3}+\frac{2}{5} \frac{R^{2}}{x^{2}}\right)\left(\begin{array}{l}
0 \\
0 \\
1
\end{array}\right) \\
& =R^{2} \Delta R \frac{\partial^{2}}{\partial x_{p} \partial x_{p}}\left(-\frac{1}{3}+\frac{1}{5} \frac{R^{2}}{r^{2}}\right) \frac{\boldsymbol{x}}{r} \\
B_{k} & =R^{2} \Delta R \Gamma \frac{\partial}{\partial x_{k}}\left(\frac{2}{3} \frac{1}{x}\right)=R^{2} \Delta R \Gamma \frac{\partial}{\partial x_{k}}\left(\frac{2}{3} \frac{1}{r}\right) .
\end{aligned}
$$

Referring back to (51), the partial differentiation is performed as follows:

$$
\frac{\partial^{2}}{\partial x_{p} \partial x_{p}}\left(\frac{x_{k}}{r}\right)=\frac{\partial^{2}}{\partial x_{p} \partial x_{p}} \frac{\partial}{\partial x_{k}} r=-\frac{2}{r^{2}} \frac{x_{k}}{r} .
$$

The other partial derivative term vanishes, as

$\frac{\partial^{2}}{\partial x_{p} \partial x_{p}}\left(\frac{1}{r^{2}} \frac{x_{k}}{r}\right)=\frac{\partial^{2}}{\partial x_{p} \partial x_{p}} \frac{\partial}{\partial x_{k}}\left(-\frac{1}{r}\right), \frac{\partial^{2}}{\partial x_{p} \partial x_{p}}\left(-\frac{1}{r}\right)=0$.

Therefore, we obtain

$$
\begin{aligned}
A_{k} & =\frac{2}{3} \Delta R\left(\frac{R}{r}\right)^{2} \frac{x_{k}}{r}, \\
B_{k} & =-\frac{2}{3} \Delta R \Gamma\left(\frac{R}{r}\right)^{2} \frac{x_{k}}{r} .
\end{aligned}
$$

Combining (72) and (73), we finally obtain

$$
u_{k}^{\mu u 1}=\frac{2}{3} \Delta R(1-\Gamma)\left(\frac{R}{r}\right)^{2} \frac{x_{k}}{r}=\frac{2}{3} \mu \frac{\Delta V^{C}}{\lambda+2 \mu} \frac{x_{k}}{4 \pi r^{2}},
$$

which cancels with $u_{k}^{\mu u 2}$ as given in (56).

\section{Competing interests}

The authors have no competing interests to declare.

\section{Authors' contributions}

All the authors contributed to discussions clarifying the theory presented in this manuscript. MI presented the idea for the new interpretation. TK solved the problem for the spherical case presented in the Appendix. NK and TK verified the mathematical consistency of the new interpretation in the representation theorem. All authors read and approved the final manuscript.

\section{Acknowledgements}

This work was motivated by the study of R. Kazahaya and Y. Maeda. The authors are grateful for M. Savage (the Editor) and the two anonymous reviewers for their constructive comments to improve the manuscript. We also thank Y. Aoki 
for valuable discussions and suggestions. M.I. was supported by JSPS (KAKENHI 24234567), T.K. was supported by a Grant-in-Aid from JSPS (No. 25-7419), and N.K. was supported by JSPS (KAKENHI 25400441). This work was also supported by MEXT of Japan via the Program for Leading Graduate Schools and by the Earthquake and Volcano Hazards Observation and Research Program.

\section{Author details}

${ }^{1}$ Earthquake Research Institute, The University of Tokyo, Yayoi, Bunkyo-ku, Tokyo 113-0032, Japan. ${ }^{2}$ Graduate School of Environmental Studies, Nagoya University, Chikusa-ku, Nagoya 464-8601, Japan.

Received: 10 September 2015 Accepted: 12 January 2016

Published online: 29 January 2016

\section{References}

Aki K, Richards PG (1980) Quantitative seismology: theory and methods. First Ed. W. H. Freeman, San Francisco

Aki, K, Richards PG (2002) Quantitative seismology. 2nd Edn. University Science Books, Sausalito

Altiero NJ, Gavazza SD (1980) On a unified boundary-integral equation method. J Elasticity 10:1-9

Amoruso A, Crescentini L (2009) Shape and volume change of pressurized ellipsoidal cavities from deformation and seismic data. J Geophys Res 114:B02210. doi:10.1029/2008JB005946

Ampuero JP, Dahlen FA (2005) Ambiguity of the moment tensor. Bull Seism Soc Am 95(2):390-400. doi:10.1785/0120040103

Backus G, Mulcahy M (1976) Moment tensors and other phenomenological descriptions of seismic sources - I. continuous displacements. Geophys J R astr Soc 46:341-361

Chouet BA (1996) New methods and future trends in seismological volcano monitoring. In: Monitoring and mitigation of volcano hazards. Springer, Berlin. pp 23-97

Davis PM (1986) Surface deformation due to inflation of an arbitrarily oriented triaxial ellipsoidal cavity in an elastic half-space, with reference to Kilauea Volcano. Hawaii J Goephys Res 91(B7):7429-7438

Eshelby JD (1957) The determination of the elastic field of an ellipsoidal inclusion and related problems. Proc R Soc London, Ser A 241:376-396

Ichihara M (2008) Dynamics of a spherical viscoelastic shell: implications to a criterion for fragmentation/expansion of bubbly magma. Earth Planet Sci Lett 265:18-21

Kame N, Kusakabe T (2012) Proposal of extended boundary integral equation method for rupture dynamics interacting with medium interfaces. J Appl Mech 79(031017). doi:10.1115/1.4005899

Kazahaya R, Mori T, Takeo M, Ohminato T, Urabe T, Maeda Y (2011) Relation between single very long period pulses and volcanic gas emissions at $\mathrm{Mt}$. Asama, Japan. Goephys Res Lett 105(B8):19,135-19,147

Kumagai H, Maeda Y, Ichihara M, Kame N, Kusakabe T (2014) Seismic moment and volume change of a spherical source. Earth Planet Space 66(7). doi:10.1186/1880-5981-66-7

Maeda Y, Takeo M (2011) Very-long-period pulses at Asama volcano, central Japan, inferred from dense seismic observations. Goephys J Int 185(1):265-282. doi:10.1111/j.1365-246X.2011.04938.x

Mizuno N, Ichihara M, Kame N (2015) Moment tensors associated with expansion and movement of fluid in ellipsoidal cavities. J Geophys Res. in press

Müller G (2001) Volume change of seismic sources from moment tensors. Bull Seism Soc Am 91:880-884

Nishimura T, Nakamichi H, Tanaka S, Sato M, Kobayashi T, Ueki S, Hamaguchi H, Ohtake M, Sato H (2000) Source process of very long period seismic events associated with the 1998 activity of Iwate Volcano, northeastern Japan. J Goephys Res 38:L11307. doi:10.1029/2011GL047555

Richards PG, Kim W-Y (2005) Equivalent volume sources for explosions at depth: theory and observations. Bull Seism Soc Am 95(2):401-407. doi:10.1785/0120040034

Wielandt E (2003) On the relationship between seismic moment and source volume. http://www.software-for-seismometry.de/textfiles/ TheoreticalSeismology/volsource.ps

\section{Submit your manuscript to a SpringerOpen ${ }^{\circ}$ journal and benefit from:}

- Convenient online submission

- Rigorous peer review

- Immediate publication on acceptance

- Open access: articles freely available online

- High visibility within the field

- Retaining the copyright to your article

Submit your next manuscript at $\gg$ springeropen.com 